\title{
Impact of sitagliptin on endometrial mesenchymal stem-like progenitor cells: A randomised, double-blind placebo-controlled feasibility trial
}

\author{
Shreeya Tewary a,b,1, Emma S. Lucas ${ }^{\mathrm{a}, \mathrm{b}, 1}$, Risa Fujihara ${ }^{\mathrm{a}, \mathrm{c}}$, Peter K. Kimani ${ }^{\mathrm{d}}$, Angela Polanco ${ }^{\mathrm{b}}$, \\ Paul J. Brighton a , Joanne Muter ${ }^{\mathrm{a}, \mathrm{b}}$, Katherine J. Fishwick ${ }^{\mathrm{a}}$, Maria José Minhoto Diniz Da Costa ${ }^{\mathrm{a}, \mathrm{b}}$, \\ Lauren J. Ewington $^{\mathrm{a}, \mathrm{b}}$, Lauren Lacey ${ }^{\mathrm{a}, \mathrm{b}}$, Satoru Takeda ${ }^{\mathrm{c}}$, Jan J. Brosens ${ }^{\mathrm{a}, \mathrm{b}}$, Siobhan Quenby ${ }^{\mathrm{a}, \mathrm{b}, *}$ \\ a Division of Biomedical Sciences, Clinical Science Research Laboratories, Warwick Medical School, University of Warwick, Coventry CV2 2DX, UK \\ b Tommy's National Centre for Miscarriage Research, University Hospitals Coventry \& Warwickshire, Coventry CV2 2DX, UK \\ c Department of Obstetrics \& Gynaecology, Juntendo University, Faculty of Medicine, 2-1-1 Hongo, Bunkyo-ku, Tokyo 113-8421, Japan \\ ${ }^{\mathrm{d}}$ Division of Health Sciences, Warwick Medical School, University of Warwick, Coventry CV4 7AL, UK
}

\section{A R T I C L E I N F O}

\section{Article History:}

Received 1 October 2019

Revised 17 November 2019

Accepted 10 December 2019

Available online $\mathrm{xxx}$

\section{Keywords:}

Pregnancy

Miscarriage

Sitagliptin

Endometrium stem cells

Randomised clinical trial

Decidualization

\begin{abstract}
A B S T R A C T
Background: Recurrent pregnancy loss (RPL) is associated with the loss of endometrial mesenchymal stem-like progenitor cells (eMSC). DPP4 inhibitors may increase homing and engraftment of bone marrow-derived cells to sites of tissue injury. Here, we evaluated the effect of the DPP4 inhibitor sitagliptin on eMSC in women with RPL, determined the impact on endometrial decidualization, and assessed the feasibility of a full-scale clinical trial. Methods: A double-blind, randomised, placebo-controlled feasibility trial on women aged 18 to 42 years with a history of 3 or more miscarriages, regular menstrual cycles, and no contraindications to sitagliptin. Thirtyeight subjects were randomised to either $100 \mathrm{mg}$ sitagliptin daily for 3 consecutive cycles or identical placebo capsules. Computer generated, permuted block randomisation was used to allocate treatment packs. Colony forming unit (CFU) assays were used to quantify eMSC in midluteal endometrial biopsies. The primary outcome measure was CFU counts. Secondary outcome measures were endometrial thickness, study acceptability, and first pregnancy outcome within 12 months following the study. Tissue samples were subjected to explorative investigations.

Findings: CFU counts following sitagliptin were higher compared to placebo only when adjusted for baseline CFU counts and age (RR: $1.52,95 \% \mathrm{CI}: 1.32-1.75, P<0.01$ ). The change in CFU count was 1.68 in the sitagliptin group and 1.08 in the placebo group. Trial recruitment, acceptability, and drug compliance were high. There were no serious adverse events. Explorative investigations showed that sitagliptin inhibits the expression of DIO2, a marker gene of senescent decidual cells.

Interpretation: Sitagliptin increases eMSCs and decreases decidual senescence. A large-scale clinical trial evaluating the impact of preconception sitagliptin treatment on pregnancy outcome in RPL is feasible and warranted. Funding: Tommy's Baby Charity.

Clinical trial registration: EU Clinical Trials Register no. 2016-001120-54.
\end{abstract}

Crown Copyright (C) 2019 Published by Elsevier B.V. This is an open access article under the CC BY-NC-ND license. (http://creativecommons.org/licenses/by-nc-nd/4.0/)

\section{Introduction}

Recurrent pregnancy loss (RPL), defined as two or more spontaneous pregnancy losses prior to foetal viability, is a devastating disorder for which there are few effective treatment options [1,2]. An estimated $5 \%$ of women will experience two consecutive miscarriages and $1 \%$ three or more losses [3]. The most common cause of sporadic

\footnotetext{
* Corresponding author at: Division of Biomedical Sciences, Warwick Medical School, University Hospitals Coventry and Warwickshire, Coventry, CV2 2DX, United Kingdom.

E-mail address: S.Quenby@warwick.ac.uk(S. Quenby).

1 These authors contributed equally.
}

(non-recurrent) pregnancy loss is foetal chromosome errors, some of which are linked to maternal age [4-6]. Although consecutive pregnancies with foetal chromosomal aberrations can account for RPL [7], the frequency of euploid miscarriage increases with each additional loss $[8,9]$. These epidemiological observations suggest that uterine factors are a major cause of higher-order miscarriages [8].

Based on an unbiased genome-wide DNA methylation analysis, we reported recently that RPL is associated with loss of a conspicuous epigenetic stem cell signature in endometrial stromal cells (EnSC) isolated from midluteal biopsies [10]. Colony-forming unit (CFU) assays confirmed a reduction in endometrial mesenchymal stem-like progenitor cells (eMSC) in RPL. Further, loss of eMSC has been linked 


\section{Research in context}

\section{Evidence before this study}

Recurrent pregnancy loss (RPL) is a prevalent disorder for which there are few effective treatment options. Loss of endometrial plasticity, defined by a reduction in endometrial mesenchymal stem-like progenitor cells (eMSC) and increased abundance of senescent decidual cells, during the midluteal window of implantation has been linked to RPL. Endometrial homoeostasis and cyclic regeneration following menstrual shedding are dependant, at least partly, on recruitment and engraftment of bone marrow-derived cells (BMDC) and their subsequent differentiation into non-hematopoietic endometrial cell lineages, including decidual cells. Dipeptidyl-peptidase IV (DPP4) inhibitors, such as sitagliptin, increase the bioavailability of stromal cell-derived factor- $1 \alpha$, a potent chemoattractant implicated in homing and engraftment of BMDC to sites of tissue injury. Whether or not sitagliptin, an oral antidiabetic drug, can be used to increase the abundance of eMSC during the implantation window in RPL patients is not known.

\section{Added value of this study}

This double-blind, randomised, placebo-controlled trial demonstrates that sitagliptin (100 mg daily) over 3 consecutive menstrual cycles is effective in increasing the abundance of clonal eMSC during the midluteal phase of the cycle. The increase in eMSC in response to sitagliptin treatment was paralleled by a decrease in the expression of $\mathrm{DIO2}$, a marker gene of senescent decidual cells. We found no evidence that sitagliptin at the concentration used inhibits endometrial DPP4 activity or impacts directly on the clonogenicity of endometrial progenitor cells. Pre-conceptual sitagliptin treatment was acceptable to RPL patients and drug compliance was high. No serious adverse events were reported.

\section{Implications of all the available evidence}

To our knowledge, this is the first evidence that endometrial plasticity during the implantation window can be enhanced pharmacologically in RPL patients. The effectiveness of preconception sitagliptin treatment in miscarriage prevention warrants further testing in a large clinical trial.

to impaired decidualization $[10,11]$. Decidualization denotes the process of intense tissue remodelling that transforms the cycling endometrium upon embryo implantation into a semi-permanent tissue, the decidua, capable of accommodating the rapidly expanding placenta in pregnancy [12]. This multi-step process, driven by the postovulatory rise in circulating progesterone and rising intracellular cyclic adenosine monophosphate levels $[13,14]$, starts with an acute stress response that leads to differentiation of EnSC into specialised decidual cells $[11,15,16]$. However, a sizeable population of EnSC fail to differentiate and emerge as acute senescent decidual cells [11]. Cellular senescence is defined by a state of permanent cell-cycle arrest and secretion of inflammatory mediators, chemokines, growth factors, and extracellular matrix (ECM) proteinases, referred to as the senescence-associated secretory phenotype (SASP) [17-19]. In chronic senescence associated with ageing, the SASP is responsible for disruption of normal tissue architecture and function through diverse mechanisms, including excessive ECM remodelling, recruitment of inflammatory immune cells, and inhibition of stem cell function [18,19]. By contrast, acute senescent cells are involved in tightly orchestrated biological processes, such as wound healing, tissue repair and embryo development, and produce a SASP with defined paracrine functions [20-22]. Also, acute senescent cells typically selforganise their elimination by recruiting innate immune cells, foremost natural killer (NK) cells [18,23,24]. These defining characteristics also apply to the midluteal endometrium. For example, the initial inflammatory decidual 'secretome' has been shown experimentally to induce expression of key endometrial receptivity genes [25]. Further, we demonstrated that decidual cells orchestrate the elimination of their senescent counterparts by secreting factors, such as interleukin 15 (IL-15) and C-X-C motif chemokine ligand 14 (CXCL14), involved in recruitment and activation of uterine NK cells [11], de facto curtailing tissue inflammation and facilitating transformation of the stroma into the decidua of pregnancy. Based on marker genes of decidual subsets identified by high-throughput single-cell RNAsequencing, we demonstrated that the midluteal endometrium in RPL is associated not only with eMSC deficiency but also excessive decidual senescence [26], an imbalance that arguably predisposes to the formation of a proinflammatory and intrinsically unstable placental-decidual interface in pregnancy.

Decidual cells but not senescent decidual cells are dependant on continuous progesterone signalling [26]. Consequently, in the absence of embryo implantation, falling ovarian progesterone production leads to a preponderance of senescent cells and activation of a cascade of events that result in tissue breakdown, bleeding and menstrual shedding of the superficial endometrial layer $[27,28]$. It is widely accepted that cyclic endometrial repair following menstrual injury involves activation of epithelial progenitor cells and eMSC residing in the basal layer [29-31]. However, bone marrow transplantation studies in humans and mice have provided compelling evidence that endometrial regeneration and homoeostasis also depend, at least partly, on recruitment and engraftment of bone marrow-derived cells (BMDC) and their subsequent differentiation into non-hematopoietic endometrial lineages, including endothelial, stromal and epithelial cells [32-36]. Stromal cell-derived factor- $1 \alpha$ (SDF1), also known as CXCL12, is a potent chemotactic factor that mediates mobilization of BMDC and homing to the endometrium in response to tissue injury and rising oestradiol levels $[37,38]$. However, SDF-1 is proteolytically inactivated by dipeptidyl-peptidase IV (DPP4), a ubiquitous aminopeptidase expressed both as a cell surface-bound protein and in soluble form [39]. DPP4 inhibitors (gliptins) are commonly used oral antidiabetic drugs for the treatment of type 2 diabetes [40]. They improve glucose homoeostasis by preventing degradation of the incretin hormones glucagon-like peptide- 1 and glucose-dependant insulinotropic polypeptide [41]. In addition, numerous preclinical studies reported that DPP4 inhibitors confer cardiovascular protection and promote tissue regeneration following injury by increasing SDF-1 bioactivity, although the results of clinical trials to date have been more ambiguous [39,40,42,43]. Gliptins have a good safety profile, do not cause hypoglycaemic episodes, and are well-tolerated in both diabetic and nondiabetic patients [40].

Based on these observations, we hypothesised that sitagliptin, given over multiple menstrual cycles, will enhance the abundance of eMSC in RPL patients. To test this hypothesis, we carried out a randomised, double-blind, placebo-controlled feasibility trial. Tissue samples obtained during the study and primary cultures were subjected to additional analysis to explore the mechanisms of sitagliptin actions in the endometrium and to assess the impact on decidual subpopulations.

\section{Materials and methods}

\subsection{Study governance}

The SIMPLANT study was approved by the Medicines and Healthcare Regulatory Authority (MHRA), the National Health Service Research Ethics Committee South Central-Hampshire B (16/SC/0229) and Research, Development and Innovation (RD\&I) office at 
University Hospitals Coventry and Warwickshire (UHCW) National Health Service (NHS) Trust. The study was sponsored by UHCW NHS Trust and funded by Tommy's baby charity (registered charity $1,060,508 /$ SC039280, Great Britain). The study protocol was submitted to the EU Clinical Trials Register (EudraCT number 2016-001120-54 issued 25th July 2016) and is available on-line (clinicaltrialsregister.eu). The date of enrolment of first participant was 15th September 2016.

\subsection{Participants}

This was a single-centre trial. Participants were recruited from a tertiary recurrent miscarriage clinic at UHCW NHS Trust. Women were eligible if aged between 18 and 42 years, had a history of 3 or more consecutive miscarriages, and regular menstrual cycles (up to 30 days in length). All participants were deemed to have unexplained miscarriages following standard RPL investigations. Participants agreed to actively avoid pregnancy and use barrier contraception for the duration of the trial. Exclusion criteria were diabetes mellitus and contraindications for the use of sitagliptin: history of pancreatitis, renal or hepatic impairment, taking digoxin or enalapril, and breastfeeding.

\subsection{Sample size}

Sample size was determined by a power calculation based on previously reported data [10]. Using a Poisson model and simulations, a sample size of 30 participants was calculated to have $91 \%$ power at $5 \%$ significance level to detect a difference when the mean CFU counts for placebo and sitagliptin groups are 3.4 and 6 per 1000 EnSC, respectively. To allow for drop-out, the planned minimum sample size was 34 women. Recruitment to the study was stopped after 30 women completed the study.

\subsection{Intervention and control groups}

The intervention group were allocated packs containing sitagliptin $(100 \mathrm{mg})$ that had been encapsulated by Sharp Clinical Services (Powys, UK). Participants were instructed to take the capsules once daily from the day of the baseline biopsy for 3 menstrual cycles until the second biopsy was taken. The control group were allocated identical placebo capsules also supplied by Sharp Clinical Services and instructed to take these exactly as the intervention group.

\subsection{Randomisation and masking}

The study statistician used the $\mathrm{R}$ statistical package to generate a randomisation list of 40 participants using the permuted block randomisation. The block sizes were $6,8,6,8,6$ and 6 . The ratio of women in the two groups within a block was 1:1. An independent statistician checked the $\mathrm{R}$ programme used to generate the randomisation list, the final randomisation lists, and the information inside all code break envelopes. Only the statisticians were unblinded to the block sizes and the master randomisation list. The statisticians were otherwise not involved in trial execution. Participants, the trial team, and laboratory staff were blinded to the medication assignment until completion of the trial. Immediately following the baseline biopsy, the clinical team contacted the RD\&I office at UHCW NHS Trust to request treatment pack number allocation for each participant. These pack numbers were then allocated to participants using the randomisation list. The study statistician prepared a sealed code break envelope for each participant, which was kept securely by the sponsor in case unblinding was required. Unblinding was not needed in the trial.

\subsection{Primary and secondary outcome measures}

The primary outcome measure was the CFU count, assessed by CFU assays, in midluteal biopsies. CFU count is a surrogate for eMSC count. The primary outcome was measured at baseline (pre-treatment) and following 3 cycles of placebo or sitagliptin (posttreatment). The pre-planned primary analysis was to compare the posttreatment CFU count per 1000 EnSC. The prespecified secondary analysis was to compare the posttreatment CFU counts adjusted for characteristics that were different between the placebo and sitagliptin groups. Secondary outcome measures not included in the protocol were endometrial thickness, study acceptability, and first pregnancy outcome within 12 months following the study. Tissue samples and primary endometrial cell cultures were used to explore the mechanisms of sitagliptin actions in the endometrium and to assess the impact on decidualization. The methods used are described below.

\subsection{Transvaginal ultrasound, endometrial biopsies and scheduled study visits}

Following written informed consent, participants were given a digital home ovulation test kit (Clearblue, Geneva, Switzerland), an emergency contact card, and barrier contraception. Participants then attended the clinic 7-10 days after the luteinizing hormone ( $\mathrm{LH})$ surge (LH+7-10). A transvaginal pelvic ultrasound scan was performed after a negative urinary pregnancy test. Endometrial thickness was defined as the maximal endometrium diameter measured in a midsagittal plane. Next, an endometrial biopsy was obtained using a Wallach Endocell ${ }^{\circledR}$ endometrial sampler. The sample, designated 'baseline biopsy', was immediately portioned with one part stored in RNALater Stabilization Solution (Sigma-Aldrich, Dorset, UK), one part snap frozen in liquid nitrogen, one part fixed in $10 \%$ formalin for immunohistochemistry, and the remainder placed in 10\% DMEM/F12 for isolation of primary EnSC (see below). Following randomisation, trial medication was provided and all participants attended two follow-up clinics at 4 weeks \pm 4 days intervals. At these follow-up visits, a urine pregnancy test was carried out, symptom diaries were reviewed, adverse events reported, and willingness for continued participation confirmed. At the second follow-up visit, participants were provided with a home ovulation test kit. In the third cycle, participants attended the clinic on LH+7-10 for a repeat transvaginal ultrasound scan with measurement of endometrial thickness and endometrial biopsy. A second endometrial sample, designated 'second biopsy', was obtained and processed identically to the baseline biopsy. Trial medication was stopped on the day of the second biopsy.

\subsection{Study acceptability, compliance, adverse events and pregnancy outcome}

Participants were provided with compliance and symptom diaries at each visit. Diaries were assessed at every follow-up or biopsy visit. Acceptability and satisfaction of the study was assessed with a Likert scale-based questionnaire completed by participants after their final visit. Participants who conceived after completion of the study were requested to contact the trial team and outcome of the first pregnancy within one year of study completion was recorded.

\subsection{Isolation of primary EnSC}

Primary EnSC were isolated according to our published protocol [44]. Briefly, samples were washed in DMEM/F-12 medium (Invitrogen), finely minced and enzymatically digested with $0.5 \mathrm{mg} / \mathrm{ml}$ collagenase (Sigma-Aldrich) and $0.1 \mathrm{mg} / \mathrm{ml}$ deoxyribonuclease type I (Roche, Burgess Hill, UK) for $1 \mathrm{~h}$ in $5 \% \mathrm{CO}_{2}$ at $37^{\circ} \mathrm{C}$. Stromal and epithelial fractions were separated and cryopreserved in 10\% DMSO in dextran-coated charcoal-stripped foetal bovine serum (DCC; $2 \mathrm{ml}$ per vial; Invitrogen), with the stromal fraction split into two or three vials depending on the biopsy size. After controlled cooling at $-80{ }^{\circ} \mathrm{C}$ overnight, samples were transferred to liquid nitrogen for storage. 


\subsection{Colony-forming unit (CFU) assay}

All CFU assays were conducted in batches, consisting of paired primary EnSC isolated from baseline and second biopsies from 3 or 4 participants. CFU assays were established as described previously [45]. Briefly, cryopreserved EnSC were thawed for $3 \mathrm{~min}$ at $37^{\circ} \mathrm{C}$ and then transferred immediately into pre-warmed growth medium (DMEMF12 medium containing 10\% DCC supplemented with 1\% L-Glutamine and $1 \%$ antibiotic-antimycotic mix; Invitrogen). Cells were pelleted by centrifugation at $276 \times g$ for $5 \mathrm{~min}$, supernatant was aspirated and cells were resuspended in $10 \mathrm{ml}$ growth medium. Viable EnSC were counted in trypan blue on a Neubauer Improved haemocytometer and seeded at clonal density (53 cells $/ \mathrm{cm}^{2}$ ) onto fibronectin-coated 6-well plates [10 $\mu \mathrm{g} / \mathrm{ml}$ in phosphate buffered saline (PBS); Sigma-Aldrich] in growth medium supplemented with $10 \mathrm{ng} / \mu \mathrm{l}$ basic fibroblast growth factor (Sigma Aldrich). Three wells, containing 500 cells each, were seeded per biopsy. Plates were cultured in $5 \% \mathrm{CO}_{2}$ at $37{ }^{\circ} \mathrm{C}$ and left undisturbed for 3 days after which growth was monitored to ensure colonies arose from single cells. The culture media was half-changed after 7 days of culture. On day 10, cultures were washed in PBS, fixed in $10 \%$ neutral buffered formalin for $10 \mathrm{~min}$ at room temperature, washed extensively in sterile water, and then stained with Harris hematoxylin for $4 \mathrm{~min}$. After extensive washing in sterile water, plates were incubated in PBS for 4 min to intensify the stain. PBS was removed and plates washed again in sterile water and then allowed to dry. Colonies were imaged using a G:Box dark room imager and GeneSys software (Syngene). Images were analysed in Image by a single operator using the cell counter plugin to count colonies of 50 cells or larger [10]. The pre-specified primary outcome measure was the CFU count per 1000 EnSC seeded after 3 cycles of sitagliptin or placebo. However, to mitigate against potential loss of data in case of infection, a total of 1500 cells were seeded in 3 wells of a 6 -well plate per sample. As there were no obvious criteria to exclude the colony count from a given well, the results are presented as CFU count per 1500 EnSC.

\subsection{DPP4 activity assay}

DPP4 activity in whole tissue lysates from snap-frozen endometrial biopsies was measured using DPP4 Activity Assay Kit (SigmaAldrich) according to the manufacturer's instructions. DPP4 activity was normalised to total protein content. The Bradford assay was used to measure protein concentrations (Sigma-Aldrich).

\subsection{Reverse transcription quantitative $P C R(R T-q P C R)$}

RNA was extracted from RNAlater-preserved tissue using the RNeasy Lipid Tissue Mini Kit (QIAGEN, Manchester, UK) with on-column DNase treatment and according to the manufacturer's instructions. RNA was assessed using a Nanodrop ND-1000 spectrophotometer and $1 \mu \mathrm{g}$ RNA used for reverse transcription using the Quantitect Reverse Transcription Kit (QIAGEN) and according to manufacturer's instructions. Thermal cycling was performed on a QuantStudio 5 Real-Time PCR System (ThermoFisher, Paisley, UK), using PrecisionPlus $2 \times$ Mastermix (Primer Design, Southampton, UK), $300 \mathrm{nM}$ forward and reverse primers, and $1 \mu \mathrm{l}$ cDNA. L19 was used as a housekeeping gene and data were analysed using the Pfaffl method [46]. Primer sequences were as follows: PRL (F) 5'-AAG CTG TAG AGA TTG AGG AGC AAA C-3', PRL (R) 5'-TCA GGA TGA ACC TGG CTG ACT A-3'; DPP4 (F) 5'-CCA AAG ACT GTA CGG GTTC C-3', DPP4 (R): 5'-ACA AAG AAC TTT ACA GTT GGA TTC AC-3'; IGFBP1 (F) 5'-CGA AGG CTC TCC ATG TCA CCA-3', IGFBP1 (R), 5-TGT CTC CTG TGC CTT GGC TAA AC-3; SCARA5 (F) 5'-CAT GCG TGG GTT CAA AGG TG-3', SCARA5 (R) 5'-CCA TTC ACC AGG CGG ATC AT-3'; DIO2 (F) 5'-ACT CGG TCA TTC TGC TCA A-3', DIO2 (R) 5'-TTC CAG ACG CAG CGC AGT-3'; L19 (F) 5'-GCG GAA GGG TAC AGC CAA T-3', L19 (R) 5'-GCA GCC GGC GCA AA-3'.

\subsection{In vitro sitagliptin experiments: DPP4 assay}

Primary cultures of EnEC or EnSC were established as described before [44]. Confluent cultures were exposed to pharmacological concentrations of sitagliptin for one hour [47]. Conditioned culture media (culture supernatant) was collected and enzymatic activity of secreted DPP4 measured using the DPP4 Activity Assay according to the manufacturer's instructions (Sigma-Aldrich).

\subsection{In vitro sitagliptin experiments: CFU and organoid forming efficiency assays}

CFU assays were performed on cultured EnSC at passage 2 in the presence or absence of $100 \mu \mathrm{M}$ sitagliptin. CFUs were fixed, stained and counted after 10 days in culture, as described in Section 2.10.

Epithelial gland organoids were established using a recently described protocol [48]. To determine the impact of sitagliptin on epithelial progenitors, EnEC were seeded at a density of 300 cells per $5 \mu \mathrm{l}$ ice cold Matrigel (Corning) in 96-well plates. Matrigel was first polymerised at $37^{\circ} \mathrm{C}$ for $45 \mathrm{~min}$ and then $100 \mu$ l organoid expansion medium, containing epidermal growth factor, fibroblast growth factor 10, hepatocyte growth factor, Noggin (BMP4 antagonist), R-spon$\operatorname{din}-1$ (WNT/ $\beta$ catenin pathway agonist), A83-01 (Alk3/4/5 inhibitor), and nicotinamide, was added and supplemented with $100 \mu \mathrm{M}$ sitagliptin or vehicle control. Medium was changed every 2 days and the number of organoids per well was counted after 10 days. At least 3 wells per sample were counted and averaged. The following formula was used to calculate organoid forming efficacy: OFE $(\%)=$ (number of organoids / cells seeded) $\times 100$.

\subsection{Statistical analysis}

Trial data were analysed using $\mathrm{R}$ [49]. Participant characteristics [age, body mass index (BMI), number of previous miscarriages] and baseline and second CFU counts were summarised as the mean, median, minimum, maximum, standard deviation and interquartile range. Mann-Whitney $U$ test was used to compare baseline characteristics between the placebo and sitagliptin groups. The primary analysis of the primary outcome fitted a Poisson regression model to compare the mean CFU count per 1500 EnSC following 3 cycles of sitagliptin or placebo. As planned in the protocol, a secondary analysis of the primary outcome adjusted for characteristics that were imbalanced between control and intervention (i.e. age and baseline CFU count) was performed. To estimate the increase from baseline, a random effects Poisson regression model that consisted of the baseline and second CFU counts was fitted. The random effects model included an interaction term for biopsy and group. An exploratory subgroup analysis excluding 8 women with substantially higher baseline CFU count ( $>20$ colonies per 1500 cells seeded) was also performed. For other secondary outcome measures, Kruskal-Wallis with Dunn's post-hoc test for multiple comparisons was used for grouped analysis (endometrial thickness) and Mann-Whitney U test was used for pairwise comparisons (LH timing). For exploratory analyses, statistical tests were performed using GraphPad Prism (version 8.2.1). Wilcoxon matched-pairs signed rank test with Sidak correction for multiple testing (alpha $=0.025$ ) was used to analyse differences in gene expression or DPP4 activity between the baseline and second biopsy in either the sitagliptin or placebo group. Temporal changes in relative gene expression or DPP4 activity between the placebo and sitagliptin groups was compared using Mann-Whitney U test. DPP4 activity in EnEC and EnSC culture supernatants were compared using Mann-Whitney U test. CFU activity and OFE in paired cultures treated with or without sitagliptin were compared using Wilcoxon matched-pairs signed rank test. Unless stated otherwise, $P<0.05$ was considered significant. 


\section{Results}

\subsection{Patient recruitment and trial completion}

The study was discussed with 73 patients with a severe RPL phenotype, defined by the number of previous miscarriages and a history of at least one euploid pregnancy loss confirmed by cytogenetic analysis. Forty-two subjects consented to the study but four were lost prior to randomisation: two withdrew consent, one participant had a urea level outside the reference range, and insufficient tissue was obtained in the baseline biopsy of one participant. A total of 19 women were allocated to each treatment group. In the sitagliptin group, 16 completed the study, one participant was withdrawn by the sponsor, one opted-out and one was lost to follow-up. In the placebo group, two participants became pregnant during the trial and 17 completed the study. The CONSORT diagram is presented in Fig. 1. The first participant consented on 15 September 2016 and the last participant to have a second biopsy attended on 16 February 2018. Pregnancy follow-up was completed on 28 February 2019.

\subsection{Patient characteristics and CFU counts}

The median number of previous miscarriages in the sitagliptin and placebo groups was 5.5 and 8 , respectively (Table 1 ), underscoring the severity of the RPL phenotype of study participants. The number of previous pregnancy losses, BMI, day of the biopsy relative to the LH-surge, and baseline CFU counts were not significantly different between the sitagliptin and placebo groups $(P<0.05$; Table 1). However, the median age of participants in the sitagliptin group was higher than in the placebo group ( 36 vs. 32 years, $P=0.02$ ) (Table 1).

The primary outcome measure was based on the CFU counts of the second biopsies upon completion of the trial. Although 33 participants completed the trial, second biopsy CFU counts were available for 32 subjects because of one case of yeast contamination in the sitagliptin group. Conversely, the baseline count was lacking for one subject in the placebo group as no viable cells were recovered. The primary outcome analysis showed no significant difference in the unadjusted mean CFU count after 3 cycles of sitagliptin compared to placebo $(P=0.15$; Table 2). When adjusted for baseline CFU count, the mean CFU count in the second biopsy was significantly higher in the sitagliptin group compared to the placebo group (27.67 vs. 25.06, RR: 1.51, $95 \% \mathrm{CI}=1.31-1.73, P<0.01)$. Adjusting for age and baseline $\mathrm{CFU}$ count had minimal impact on results (Table 2). Unadjusted subgroup analysis that excluded 8 outlying CFU counts ( $>20$ colonies per 1500 cells) also demonstrated significantly higher CFU counts following sitagliptin treatment compared to placebo (14.42 vs. 11.00 , RR: $1.31,95 \% \mathrm{CI}$ $=1.04-1.63, P=0.02$ ). As shown in Fig. 2 , CFU count in the second biopsy was significantly higher when compared to the baseline biopsy in the sitagliptin arm (median increase in colonies: +7 per 1500 cells, 1.68 -fold the baseline count, $P<0.01$ ). By contrast, there was no significant increase in the CFU count between the baseline and second biopsy in the placebo group (median increase:-0.5 per 1500 cells, 1.08 -fold the baseline count, $P=0.26$ ) (Fig. 2 . and Table 3 ).

\section{CONSORT Flow Diagram}

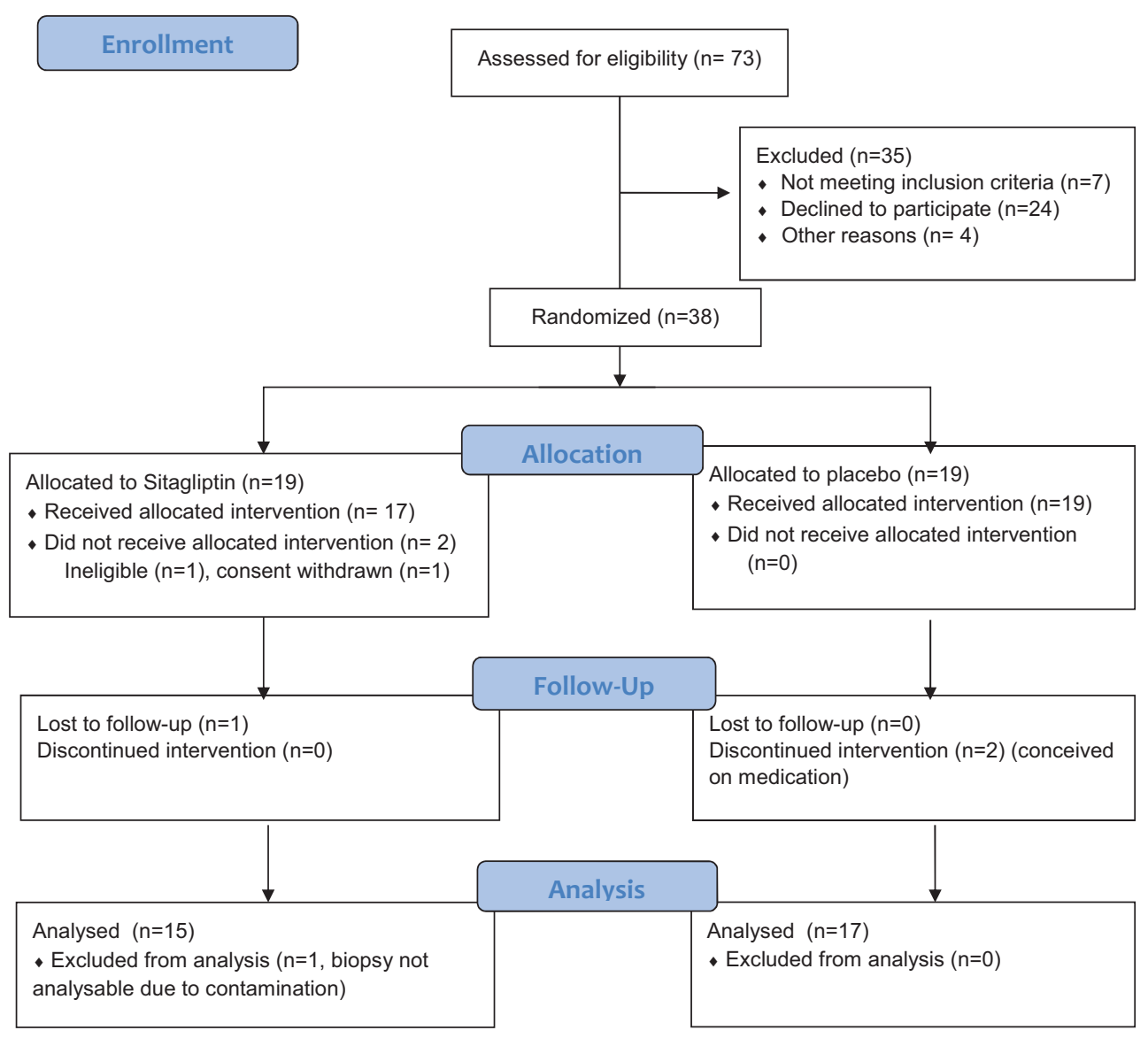

Fig. 1. Consort flow diagram. 
Table 1

Patient demographics and CFU counts at baseline and second biopsy.

\begin{tabular}{|c|c|c|c|}
\hline & $\begin{array}{l}\text { Sitagliptin Group } \\
(n=16)\end{array}$ & $\begin{array}{l}\text { Placebo Group } \\
(n=17)\end{array}$ & $P$-value ${ }^{\dagger}$ \\
\hline \multicolumn{4}{|l|}{ Age (years) } \\
\hline Median (IQR, Range) & $\begin{array}{l}36.0(31.3-38.0 \\
26-40)\end{array}$ & $\begin{array}{l}32.0(29.0-33.5 \\
24-36)\end{array}$ & 0.02 \\
\hline \multicolumn{4}{|l|}{ BMI } \\
\hline Median (IQR, Range) & $\begin{array}{c}27.3(22.4-30.3 \\
19.5-35.7)\end{array}$ & $\begin{array}{l}25.6(22.8-27.8 \\
21.1-38.4)\end{array}$ & 0.53 \\
\hline \multicolumn{4}{|l|}{$\begin{array}{l}\text { Number of } \\
\text { miscarriages }\end{array}$} \\
\hline Median (IQR, Range) & $5.5(5.0-7.0,3-14)$ & $8.0(5.0-9.5,3-14)$ & 0.36 \\
\hline \multicolumn{4}{|c|}{ Baseline CFU count } \\
\hline Median (IQR, Range) & $8.5(4.5-17.8,1-74)$ & $\begin{array}{l}12.0(6.3-45.8, \\
2-78)^{\ddagger}\end{array}$ & 0.49 \\
\hline Mean (SD) & $16.1(19.6)$ & $24.2(25.6)$ & \\
\hline \multicolumn{4}{|l|}{$\begin{array}{l}\text { Second biopsy CFU } \\
\text { count }\end{array}$} \\
\hline Median (IQR, Range) & $\begin{array}{l}14.0(8.0-29.0 \\
3-145)^{\ddagger}\end{array}$ & $\begin{array}{l}11.0(5.5-37.5 \\
3-98)\end{array}$ & 0.65 \\
\hline Mean (SD) & $27.7(35.8)$ & $25.1(27.3)$ & \\
\hline \multicolumn{4}{|l|}{$\begin{array}{l}\text { Timing of baseline } \\
\text { biopsy }\end{array}$} \\
\hline Median (IQR, Range) & $7.0(7.0-8.0)^{*}$ & $7.5(7.0-8.0)$ & 0.99 \\
\hline \multicolumn{4}{|c|}{$\begin{array}{l}\text { Timing of second } \\
\text { biopsy }\end{array}$} \\
\hline Median (IQR, Range) & $8.0(7.0-9.0)^{*}$ & $7.5(7.0-8.8)$ & 0.78 \\
\hline
\end{tabular}

\subsection{Endometrial thickness, study acceptability, and subsequent pregnancy outcome}

The median endometrial thickness at baseline was $9.0 \mathrm{~mm}$ [interquartile range (IQR): $8.5-10.0 \mathrm{~mm}$ ] and $8.9 \mathrm{~mm}$ (IQR: $7.2-9.7 \mathrm{~mm}$ ) in the placebo and sitagliptin groups, respectively. Median endometrial thickness at the time of the second biopsy was $9.6 \mathrm{~mm}$ (IQR: $7.7-10.3 \mathrm{~mm}$ ) and $8.0 \mathrm{~mm}$ (IQR: $7.2-10.6 \mathrm{~mm}$ ) in the placebo and sitagliptin groups, respectively. Hence, endometrial thickness was within the expected midluteal range for all participants and not significantly different between the placebo and sitagliptin groups, either at baseline or at the time of the second biopsy $(P>0.05$, KruskalWallis test).

Compliance to study medication was high. Out of 33 participants who completed the study, 32 demonstrated near full compliance (>98\%). Study medication compliance was $50 \%$ for one participant randomised to placebo. A questionnaire with Likert scale responses and space for free text comments showed that all participants agreed or agreed strongly that taking part in the study was worthwhile, would recommend the study to others, and found that taking the medication was easy. The only adverse event reported by more than one participant was headache, which occurred in 7 and 4 participants in the placebo and sitagliptin group, respectively; and therefore not attributable to study medication. Supplementary Table S1 lists all reported side-effects.

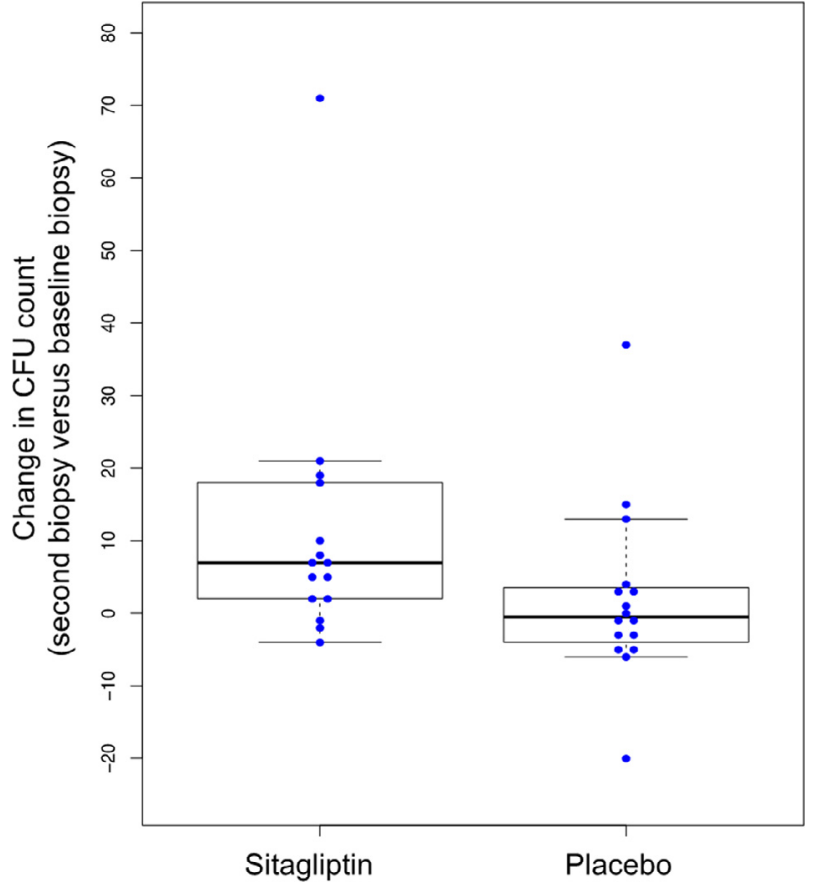

Fig. 2. Change in CFU count after 3 cycles of sitagliptin or placebo. The median increase in CFU count in the second compared to the baseline biopsy was +7 per 1500 EnSC and -0.5 per 1500 EnSC in the sitagliptin and placebo group, respectively $(P<0.01)$. Boxplots present the median, upper and lower quartiles with whiskers calculated using Tukey's method.

We recorded the outcome of the first pregnancy within 12 months following completion of the study. Out of 33 participants who completed the study, 25 reported a pregnancy outcome within this timeframe. In the sitagliptin group, there were 8 live births, one termination of pregnancy at 16 weeks for a foetal abnormality (cerebellar agenesis), and 3 spontaneous pregnancy losses before 12 weeks of gestation. Cytogenetic analysis was performed in 2 of 3 miscarriage cases and both showed foetal aneuploidy (trisomy 22 and triploidy). In the placebo group, there were 7 live births and 6 spontaneous pregnancy losses before 12 weeks. Cytogenetic analysis was performed in one case and showed normal foetal karyotype. It is important to note that this study was not powered to assess the effect of pre-pregnancy sitagliptin treatment on pregnancy outcomes.

\subsection{Explorative investigations: oral sitagliptin inhibits decidual senescence but not endometrial DPP4 activity}

DPP4 is a known marker of glandular differentiation during the midluteal phase of the cycle [50]. DPP4 is also a widely used endometrial receptivity marker gene [51]. To explore the mechanisms of sitagliptin actions in the endometrium, we first measured DPP4 activity in paired snap-frozen baseline and second biopsies from individual participants in the placebo group $(n=15)$ and sitagliptin group $(n=15)$. In addition, paired placebo $(n=16)$ and sitagliptin $(n=16)$

Table 2

CFU count after 3 cycles of sitagliptin or placebo (second biopsy).

\begin{tabular}{|c|c|c|c|c|c|}
\hline & \multicolumn{2}{|c|}{ Mean CFU count ${ }^{\dagger}$} & \multicolumn{3}{|c|}{ Rate ratio (95\% Confidence interval), $P$ value } \\
\hline & Sitagliptin & Placebo & Unadjusted analysis & Adjusted for baseline count & Adjusted for baseline count and age* \\
\hline All data & 27.7 & 25.1 & $1.10(0.96,1.26), P=0.15^{\ddagger}$ & $1.51(1.31,1.73), P<0.01$ & $1.52(1.32,1.75), P<0.01$ \\
\hline Subgroup data $^{\dagger}$ & 14.4 & 11.0 & $1.31(1.04,1.67), P=0.02$ & $1.43(1.13,1.80), P<0.01$ & $1.67(1.29,2.17), P<0.01$ \\
\hline
\end{tabular}


Table 3

Results of the random effects adjusted Poisson regression model.

\begin{tabular}{llllll}
\hline & \multicolumn{2}{c}{$\begin{array}{c}\text { Rate ratio: second to baseline biopsy } \\
(95 \% \text { confidence interval), } P \text { value }\end{array}$} & & & \multicolumn{2}{c}{$\begin{array}{c}\text { Rate ratio: sitagliptin to placebo } \\
\text { (95\% confidence interval), } P \text { value }\end{array}$} \\
\cline { 2 - 3 } & Sitagliptin & Placebo & & Baseline & Second biopsy \\
\hline All data & $1.68(1.44,1.97), P<0.01$ & $1.08(0.94,1.24), P=0.26$ & & $0.97(0.71,1.31), P=0.82$ & $1.49(1.12,2.00), P<0.01$ \\
Subgroup $^{\dagger}$ & $1.88(1.47,2.41), P<0.01$ & $1.27(0.97,1.66), P=0.08$ & & $1.01(0.71,1.43), P=0.95$ & $1.49(1.09,2.04), P<0.01$ \\
\hline
\end{tabular}

$\dagger$ Excludes data from participants with high baseline CFU ( $>20$ colonies per 1500 EnSC).
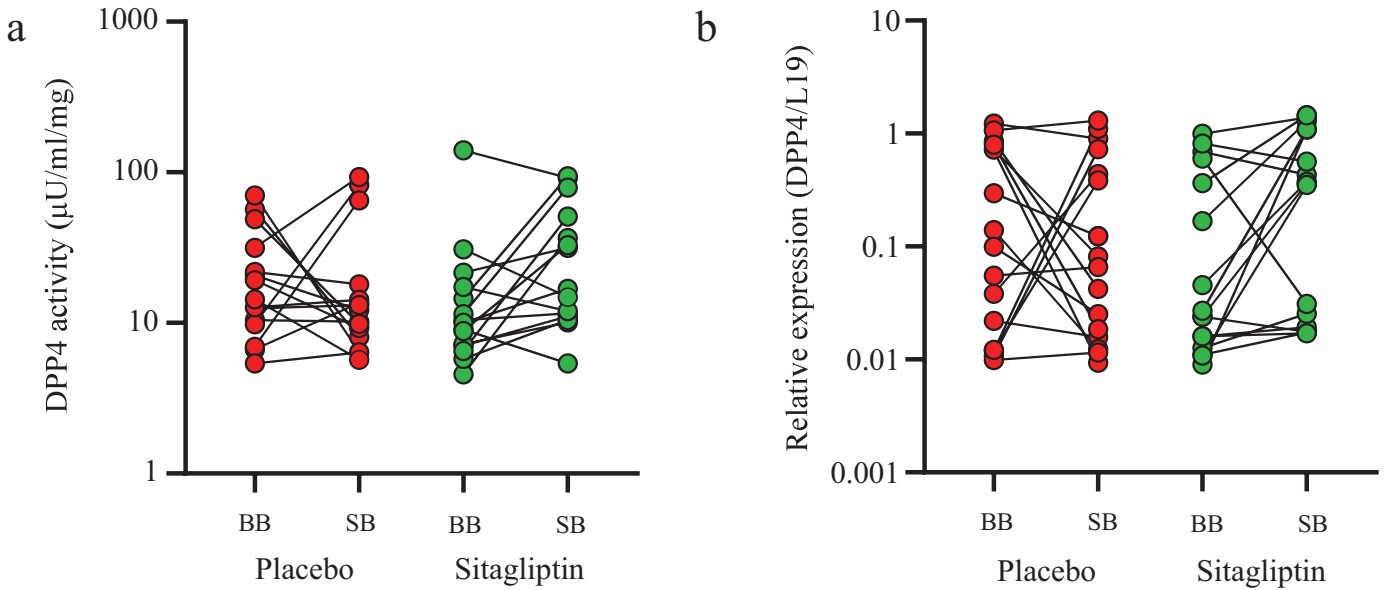

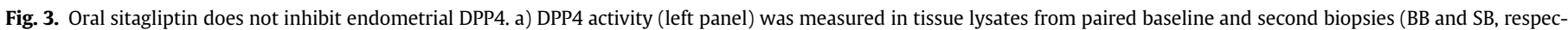

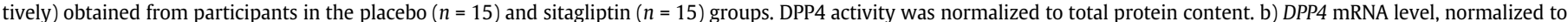

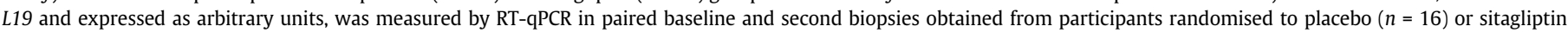
$(n=16)$. DPP4 expression and activity were not significantly different between the groups $(P>0.025$, Wilcoxon Matched Pairs Signed Ranks test with Sidak correction).

samples preserved in RNAlater were used to measure DPP4 mRNA levels by RT-qPCR. As shown in Fig. 3 and Supplementary Table S3, endometrial DPP4 activity and expression were not different between the baseline and second biopsies in the placebo or sitagliptin group $(P>0.025$, Wilcoxon test), indicating that the dose of sitagliptin (100 mg daily) was insufficient to inhibit DPP4 activity at tissue level.

We also explored if sitagliptin impacts on the clonogenicity of primary EnSC or EnEC isolated from independent midluteal biopsies. As shown in Supplementary Figure S1A, DPP4 activity was 3-fold higher in primary EnEC when compared to EnSC $(P=0.003$, Mann-Whitney U test). Exposure of primary EnEC or EnSC to pharmacological concentrations of sitagliptin inhibited DPP4 activity by 90\% (Suppl. Fig S1B). Next, we performed CFU assays on cultured EnSC seeded at very low cell density in the presence or absence of $100 \mu \mathrm{M}$ sitagliptin for 10 days. No difference in colony-forming efficiency of EnSC was observed between cultures treated with or without sitagliptin (Suppl. Fig S1C; $P>0.05$, Wilcoxon test). To test the impact on epithelial progenitor cells, we made use of a recently published protocol that enables formation of gland organoids from single endometrial epithelial progenitor cells [48]. Again, addition of sitagliptin to the expansion medium had no impact on endometrial gland organoid forming efficiency compared to control cultures (Suppl. Fig S1D; $P>0.05$, Wilcoxon test).

Next, we examined the expression of decidual marker genes in paired baseline and second biopsies from the placebo $(n=16)$ and sitagliptin $(n=16)$ groups. Induction of PRL and IGFBP1 expression is widely used to monitor the decidual response in cultured EnSC [12]. However, these marker genes do not discriminate between decidual cells and senescent decidual cells [26]. By contrast, SCARA5 and DIO2 are marker genes of decidual cells and senescent decidual cells, respectively, both in vitro and in vivo [26]. PRL and IGFBP1 transcript levels were not significantly different in the second biopsy compared to the baseline biopsy in either the placebo or treatment group (Suppl. Fig. S2 and Table S3, $P>0.025$, Wilcoxon test). By contrast, sitagliptin treatment but not placebo resulted in a significant reduction in mean DIO2 mRNA levels in the second biopsy compared to the baseline biopsy (Suppl. Table S3 and Fig. 4A, left panel [median: 0.96 arbitrary units (a.u.) vs. 3.26 a.u., respectively, $P=0.0182$, Wilcoxon test], whereas SCARA5 mRNA levels were not altered significantly (Suppl. Table S3 and Fig. 4A, right panel; $P>0.025$ ). The average reduction in $\mathrm{DIO} 2$ expression in paired biopsies was $44.4 \%$ (range: $-91.5 \%$ to $+26 \%$ ). Thus, sitagliptin treatment not only increases the abundance of eMSCs in the endometrium but also rebalances the relative abundance of decidual cell subpopulations, as measured by the fold-change in SCARA5/DIO2 ratio (Fig. 4B), by attenuating decidual senescence.

\section{Discussion}

This study reports on the feasibility of using oral sitagliptin (100 mg daily) to increase the abundance of eMSC in RPL patients. The primary outcome of this randomised, double-blind, placebo-controlled trial was CFU counts after 3 cycles of sitagliptin or placebo. Unadjusted analysis showed no statistically significant difference in CFU counts between the groups but this was accounted for by the unanticipated magnitude of interpatient variation in CFU counts. This variability in CFU counts also suggests that the severity of the RM phenotype is not solely attributable to loss of eMSC. Baseline CFU counts ranged from 1 to 78 colonies per 1500 cells seeded $(0.07 \%$ to $5.2 \%$, respectively), although only 8 subjects had CFU counts of more than 20 colonies per 1500 cells ( $>1.3 \%$ ). When adjusted for baseline CFU count, the mean CFU count in the second biopsy was $51 \%$ higher in the sitagliptin group compared to the placebo group. Compared to the baseline biopsy, sitagliptin given for 3 menstrual cycles increased CFU counts on average by $68 \%$. By contrast, there was no significant change in CFU counts in the placebo group, attesting to the robustness of the endometrial response to oral sitagliptin. The lack of a discernible effect in the placebo group demonstrated that the biopsy procedure per se had no sustained impact on the abundance of eMSC. Notably, participants randomised to the sitagliptin treatment were 
a

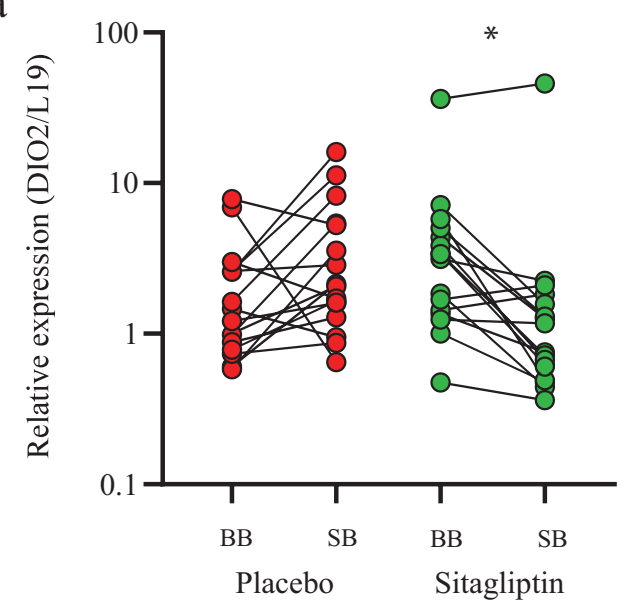

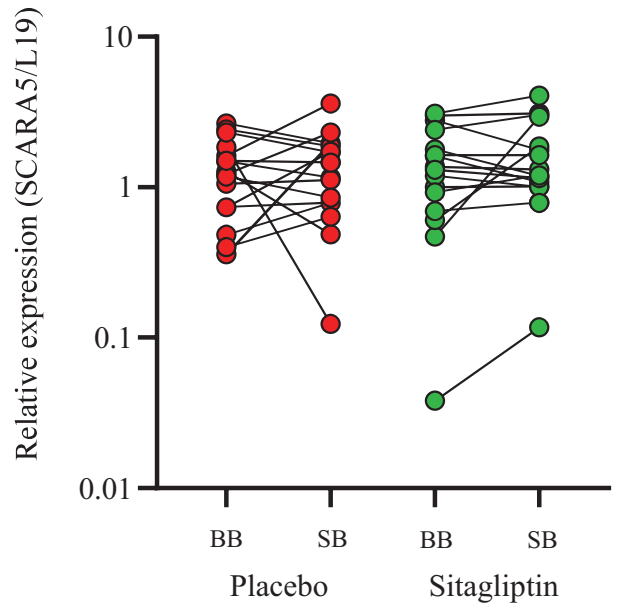

b

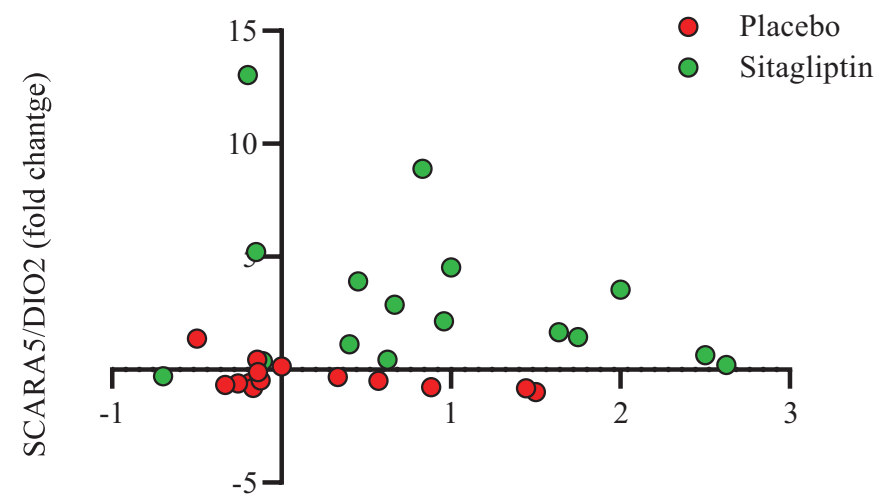

CFU (fold change)

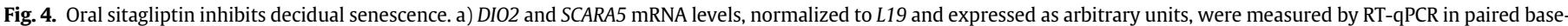

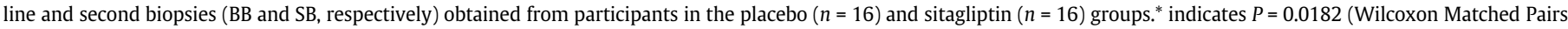

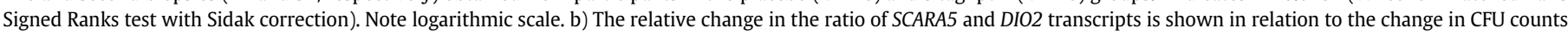
in paired endometrial biopsies from the placebo (red dots) and sitagliptin (green dots) groups.

older when compared to the placebo group. However, in line with a previous study [52], we found no evidence of a significant impact of age on either baseline CFU counts or on the response to sitagliptin. Explorative investigations indicated that the increase in eMSC in response to sitagliptin is biologically meaningful and associated with a significant reduction in senescent decidual cells. This conclusion is based on expression analysis of DIO2 and SCARA5, which are marker genes of decidual cells and senescent decidual cells, respectively [26]. DIO2 encodes iodothyronine deiodinase 2 , which catalyses the conversion of prohormone thyroxine (T4) into bioactive triiodothyronine (T3). This gene is repressed by progesterone in decidual cells but not in progesterone-resistant senescent decidual cells [26,53]. By contrast, SCARA5, coding the L-ferritin receptor (scavenger receptor class A member 5), is a progesterone-dependant marker gene of decidual cells [26,54]. Sitagliptin given over 3 cycles selectively reduced endometrial DIO2 mRNA expression by an average of $44.4 \%$, signifying a reduction in senescent decidual cells during the implantation window. Taken together, these observations indicate that pre-pregnancy sitagliptin improves the peri-implantation endometrium by simultaneously increasing tissue plasticity and decreasing decidual senescence.

Oral sitagliptin (100 mg daily) did not inhibit uterine DPP4 expression or activity. We also found no evidence that sitagliptin impacts directly on the clonal capacity of eMSCs or organoid formation efficacy of epithelial progenitor cells. Taken together, our exploratory observations are compatible with the hypothesis that oral sitagliptin inhibits DPP4-dependant inactivation of SDF-1 in the circulation [55,56]; and that homing and engraftment of BMDC in cycling endometrium is dependant on the level of circulating bioactive SDF-1 $[32,34,36]$.

The ability of gliptins to enhance regeneration of damaged tissues has been explored in other organs but by and large the clinical results have been disappointing. For example, intracoronary administration of BMDC has been shown to improve recovery of left ventricular contractile function in patients with acute myocardial infarction [57], whereas a combination of granulocyte-colony stimulating factor (GCSF)-dependant mobilisation of BMDC followed by sitagliptin treatment (100 mg for 28 days) failed to improve cardiac function [58]. However, a particular advantage of the endometrium over other tissues, such as heart or kidney, is that DPP4 inhibitors can be initiated prior or during (menstrual) tissue injury and sustained over multiple cycles. Our feasibility trial also demonstrated the acceptability of prepregnancy sitagliptin treatment in RPL patients. Drug compliance was high and no adverse events were reported. Although the trial was not designed or powered to assess to assess pregnancy outcome, only 3 spontaneous miscarriages were reported out of 12 pregnancies in the sitagliptin group, two of which were found to be caused by foetal aneuploidies.

A pharmacological approach to increase endometrial progenitor populations could be useful in the management of other intractable reproductive disorders. For example, in a murine model of thin 
endometrium, tissue regeneration and pregnancy rates were increased following treatment with either BMDC or SDF-1 [38]. A recent non-controlled study reported that autologous cell therapy in conjunction with hormonal replacement therapy temporarily improves endometrium thickness, as well as the volume and duration of menses, in patients with refractory Asherman's syndrome or endometrial atrophy [59]. This approach requires mobilization of BMDC using G-CSF, isolation of $\mathrm{CD}_{133^{+}}$endothelial progenitors through peripheral blood aphaeresis, and finally delivery of cells into the spiral arterioles by catheterization under angiography. Although we found no evidence that sitagliptin increases endometrial thickness during the luteal phase in this study, it should be noted that none of the participants had an abnormally thin midluteal endometrium.

\subsection{Limitations of the study}

A drawback of the study was the reliance on an indirect measure of eMSC, i.e. CFU assays that take 10 days to complete. As aforementioned, the interpatient variation in this assay was greater than anticipated. Also, the analysis of decidual subpopulations was based on RT-qPCR measurements of marker genes. While a recent study demonstrated that endometrial SCARA5 and DIO2 expression differs between control subjects and RPL patients [26], their value in predicting pregnancy outcome has not yet been tested. The scope of our explorative investigations was limited and the impact of oral sitagliptin on other constituents of the endometrium, such as endothelial cells and EnEC, warrants further investigation. Another limitation of the study is that it did not examine if the endometrial response was transient or sustained following cessation of treatment. Further, at least 11 different DPP4 inhibitors have now been approved for the management of type-2 diabetes worldwide [60]. Whether or not all these preparations are equivalent in terms of the endometrial response requires further investigation. As articulated before, this feasibility trial was not powered to assess the effect of pre-pregnancy sitagliptin treatment on live birth rates in RPL patients.

\subsection{Patient selection and drug dosing and timing considerations}

Our study raises a number of issues that should be considered in future studies. The first issue relates to selection of RPL patients most likely to benefit from treatment with sitagliptin. Ideally, recruitment in future studies should be based on pre-pregnancy screening for eMSC deficiency and/or excessive decidual senescence. However, it is impractical to use CFU assays in large-scale clinical studies and the use of decidual marker genes for screening purposes requires further validation. Alternatively, patients could be selected on basis of risk factors associated with euploid pregnancy loss, including the number of previous miscarriages [8,9], a prior history of euploid pregnancy loss [6], and obesity [61]. Second, in this study we opted for continuous sitagliptin treatment over 3 cycles based on the assumption that the effect on eMSC may be cumulative over multiple menstrual 'injuries'. It is possible that homing and engraftment of BMDC in the endometrium is physiologically restricted to the proliferative phase of the cycle. This conjecture is supported by murine studies demonstrating that oestradiol coordinates the induction of SDF-1 expression in EnSC with the expression of the SDF-1 receptor, $\mathrm{C}-\mathrm{X}-\mathrm{C}$ motif chemokine receptor 4 (CXCR4), in BMDC [37]. The National Institute for Health and Care Excellence (NICE) in the UK advises against the use of sitagliptin in pregnancy, a risk that could be avoided by restricting sitagliptin treatment to the proliferative phase, although the efficacy of this approach requires further evaluation. Another consideration relates to the optimal dose of sitagliptin for the purpose of miscarriage prevention in RPL. In this study, we used $100 \mathrm{mg}$ of sitagliptin daily, which is the recommended dose for glycaemic control in type 2 diabetes [62]. Higher doses of sitagliptin purportedly result in more sustained plasma DPP4 inhibition [63], which conceivably could further enhance or accelerate the engraftment of BMDC in the endometrium.

\subsection{Conclusions}

Sitagliptin increases the abundance of eMSC in midluteal endometrium and reduces decidual senescence. A large-scale clinical trial evaluating the impact of preconception sitagliptin treatment on pregnancy outcome in RPL patients is feasible and warranted.

\section{Data sharing}

All of the individual de-identified participant data and study protocol that underlie the results reported in this article (text, tables, figures, and appendices) can be made available to investigators whose proposed use of the data has been approved by an independent review committee ("learned intermediary") identified for this purpose and to achieve aims in the approved proposal, beginning 3 months and ending 5 years following publication. Proposals should be directed at S.Quenby@warwick.ac.uk.

\section{Declaration of competing interest}

Dr Brosens reports grants from Tommy's Baby Charity, grants from Juntendo University, Japan, during the conduct of the study. In addition, Dr Brosens has filed a UKIPO patent application (no.1911947.8) pertaining to the use of SCARA5 and DIO2 as endometrial biomarkers to assess the risk of miscarriage. Dr Quenby reports grants from Tommy's baby Charity, during the conduct of the study. The other authors have nothing to disclose.

\section{Funding sources}

The study was funded by Tommy's Baby Charity, United Kingdom. R.F. received funding from Juntendo University, Japan. The study was supported by the National Institute of Health Research (NIHR) Coventry and Warwickshire Clinical Research Facility. The funders had no role in: study design; in collection, analysis or interpretation of data; in the writing of the report; or in the decision to submit the paper for publication.

\section{Acknowledgements}

We are indebted to all the women whom participated in this study. We also thank all the staff members of University Hospital Coventry and Warwickshire NHS Trust and Warwick Medical School involved in the governance and execution of the study.

\section{Supplementary materials}

Supplementary material associated with this article can be found in the online version at doi:10.1016/j.ebiom.2019.102597.

\section{References}

[1] The ESHRE Guideline Group on RPL, Bender Atik R, Christiansen O, Elson J, Kolte AM, Lewis S, Middeldorp S, Nelen W, Peramo B, Quenby S, Vermeulen N, Goddijn M, ESHRE guideline: recurrent pregnancy loss, Hum Reprod Open; 2018;2:2399-3519

[2] Practice Committee of the American Society for Reproductive M. Evaluation and treatment of recurrent pregnancy loss: a committee opinion. Fertil Steril 2012;98 (5):1103-11.

[3] Rai R, Regan L. Recurrent miscarriage. Lancet 2006;368(9535):601-11.

[4] Hardy K, Hardy PJ, Jacobs PA, Lewallen K, Hassold TJ. Temporal changes in chromosome abnormalities in human spontaneous abortions: results of 40 years of analysis. Am J Med Genet A 2016;170(10):2671-80.

[5] Hassold T, Chen N, Funkhouser J, Jooss T, Manuel B, Matsuura J, et al. A cytogenetic study of 1000 spontaneous abortions. Ann Hum Genet 1980;44(2):151-78. 
[6] Stephenson MD, Awartani KA, Robinson WP. Cytogenetic analysis of miscarriages from couples with recurrent miscarriage: a case-control study. Hum Reprod 2002;17(2):446-51.

[7] Hassold TJ. A cytogenetic study of repeated spontaneous abortions. Am J Hum Genet 1980;32(5):723-30.

[8] Ogasawara M, Aoki K, Okada S, Suzumori K. Embryonic karyotype of abortuses in relation to the number of previous miscarriages. Fertil Steril 2000;73(2):300-4.

[9] Robberecht C, Pexsters A, Deprest J, Fryns JP, D'Hooghe T, Vermeesch JR. Cytogenetic and morphological analysis of early products of conception following hystero-embryoscopy from couples with recurrent pregnancy loss. Prenat Diagn 2012;32(10):933-42.

[10] Lucas ES, Dyer NP, Murakami K, Lee YH, Chan YW, Grimaldi G, et al. Loss of endometrial plasticity in recurrent pregnancy loss. Stem Cells 2016;34(2):346-56.

[11] Brighton PJ, Maruyama Y, Fishwick K, Vrljicak P, Tewary S, Fujihara R, et al. Clearance of senescent decidual cells by uterine natural killer cells in cycling human endometrium. Elife 2017:6.

[12] Gellersen B, Brosens JJ. Cyclic decidualization of the human endometrium in reproductive health and failure. Endocr Rev 2014;35(6):851-905.

[13] Brosens JJ, Hayashi N, White JO. Progesterone receptor regulates decidual prolactin expression in differentiating human endometrial stromal cells1. Endocrinology 1999;140(10):4809-20.

[14] Jones MC, Fusi L, Higham JH, Abdel-Hafiz H, Horwitz KB, Lam EW, et al. Regulation of the SUMO pathway sensitizes differentiating human endometrial stromal cells to progesterone. Proc Natl Acad Sci USA 2006:103(44):16272-7.

[15] Al-Sabbagh M, Fusi L, Higham J, Lee Y, Lei K, Hanyaloglu AC, et al. NADPH oxidasederived reactive oxygen species mediate decidualization of human endometrial stromal cells in response to cyclic AMP signaling. Endocrinology 2011;152 (2):730-40.

[16] Erkenbrack EM, Maziarz JD, Griffith OW, Liang C, Chavan AR, Nnamani MC, et al. The mammalian decidual cell evolved from a cellular stress response. PLoS Biol 2018;16(8):e2005594.

[17] Acosta JC, O'Loghlen A, Banito A, Guijarro MV, Augert A, Raguz S, et al. Chemokine signaling via the CXCR2 receptor reinforces senescence. Cell 2008;133(6):1006-18.

[18] van Deursen JM. The role of senescent cells in ageing. Nature 2014;509 (7501):439-46

[19] van Deursen JM. Senolytic therapies for healthy longevity. Science 2019;364 (6441):636-7.

[20] Jun JI, Lau LF. Cellular senescence controls fibrosis in wound healing. Aging (Albany NY) 2010;2(9):627-31.

[21] Krizhanovsky V, Yon M, Dickins RA, Hearn S, Simon J, Miething C, et al. Senescence of activated stellate cells limits liver fibrosis. Cell 2008;134(4):657-67.

[22] Storer M, Mas A, Robert-Moreno A, Pecoraro M, Ortells MC, Di Giacomo V, et al. Senescence is a developmental mechanism that contributes to embryonic growth and patterning. Cell 2013;155(5):1119-30.

[23] Antonangeli F, Zingoni A, Soriani A, Santoni A. Senescent cells: living or dying is a matter of NK cells. J Leukoc Biol 2019;105(6):1275-83.

[24] Sagiv A, Burton DG, Moshayev Z, Vadai E, Wensveen F, Ben-Dor S, et al. NKG2D ligands mediate immunosurveillance of senescent cells. Aging (Albany NY) 2016;8(2):328-44.

[25] Salker MS, Nautiyal J, Steel JH, Webster Z, Sucurovic S, Nicou M, et al. Disordered IL-33/ST2 activation in decidualizing stromal cells prolongs uterine receptivity in women with recurrent pregnancy loss. PLoS ONE 2012;7(12):e52252.

[26] Lucas ES, Vrljicak P, Muter J, Diniz-da-Costa M.M, Brighton PJ, Kong CS, Lipecki J, Fishwick K, Odendaal J, Ewington LJ, Quenby S, Ott S, Brosens J. Recurrent pregnancy loss is associated with a pro-senescence decidual response during the periimplantation window. Preprint at https://www.biorxiv.org/content/10.1101/ 368829v2.

[27] Evans J, Salamonsen LA. Inflammation, leukocytes and menstruation. Rev Endocr Metab Disord 2012;13(4):277-88.

[28] Evans J, Salamonsen LA. Decidualized human endometrial stromal cells are sensors of hormone withdrawal in the menstrual inflammatory cascade. Biol Reprod 2014;90(1):14.

[29] Gargett CE, Schwab KE, Deane JA. Endometrial stem/progenitor cells: the first 10 years. Hum Reprod Update 2016;22(2):137-63.

[30] Masuda H, Matsuzaki Y, Hiratsu E, Ono M, Nagashima T, Kajitani T, et al. Stem celllike properties of the endometrial side population: implication in endometrial regeneration. PLoS ONE 2010;5(4):e10387.

[31] Padykula HA, Coles LG, Okulicz WC, Rapaport SI, McCracken JA, King Jr. NW, et al. The basalis of the primate endometrium: a bifunctional germinal compartment. Biol Reprod 1989;40(3):681-90.

[32] Du H, Taylor HS. Contribution of bone marrow-derived stem cells to endometrium and endometriosis. Stem Cells 2007;25(8):2082-6.

[33] Gil-Sanchis C, Cervello I, Khurana S, Faus A, Verfaillie C, Simon C. Contribution of different bone marrow-derived cell types in endometrial regeneration using an irradiated murine model. Fertil Steril 2015;103(6) 1596-605 e1.

[34] Morelli SS, Rameshwar P, Goldsmith LT. Experimental evidence for bone marrow as a source of nonhematopoietic endometrial stromal and epithelial compartment cells in a murine model. Biol Reprod 2013;89(1):7.

[35] Santamaria X, Mas A, Cervello I, Taylor H, Simon C. Uterine stem cells: from basic research to advanced cell therapies. Hum Reprod Update 2018;24(6):673-93.
[36] Taylor HS. Endometrial cells derived from donor stem cells in bone marrow transplant recipients. JAMA 2004:292(1):81-5.

[37] Wang X, Mamillapalli R, Mutlu L, Du H, Taylor HS. Chemoattraction of bone marrow-derived stem cells towards human endometrial stromal cells is mediated by estradiol regulated CXCL12 and CXCR4 expression. Stem Cell Res 2015;15(1): $14-22$.

[38] Yi KW, Mamillapalli R, Sahin C, Song J, Tal R, Taylor HS. Bone marrow-derived cells or C-X-C motif chemokine 12 (CXCL12) treatment improve thin endometrium in a mouse model. Biol Reprod 2018.

[39] Zhong J, Rajagopalan S. Dipeptidyl peptidase-4 regulation of SDF-1/CXCR4 axis: implications for cardiovascular disease. Front Immunol 2015;6:477.

[40] Deacon CF. A review of dipeptidyl peptidase-4 inhibitors. Hot topics from randomized controlled trials. Diabetes Obes Metab. 2018;20 Suppl 1:34-46.

[41] Kieffer TJ, McIntosh CH, Pederson RA. Degradation of glucose-dependent insulinotropic polypeptide and truncated glucagon-like peptide 1 in vitro and in vivo by dipeptidyl peptidase IV. Endocrinology 1995;136(8):3585-96.

[42] Mulvihill EE, Drucker DJ. Pharmacology, physiology, and mechanisms of action of dipeptidyl peptidase-4 inhibitors. Endocr Rev 2014;35(6):992-1019.

[43] Xie W, Song X, Liu Z. Impact of dipeptidyl-peptidase 4 inhibitors on cardiovascular diseases. Vascul Pharmacol 2018;109:17-26.

[44] Barros FS, Brosens JJ, Brighton PJ. Isolation and primary culture of various cel types from whole human endometrial biopsies. Bio Protoc 2016;6:e2028.

[45] Masuda H, Anwar SS, Buhring HJ, Rao JR, Gargett CE. A novel marker of human endometrial mesenchymal stem-like cells. Cell Transplant 2012;21(10):2201-14.

[46] Pfaffl MW. A new mathematical model for relative quantification in real-time RTPCR. Nucleic Acids Res 2001;29(9):e45

[47] Sangle GV, Lauffer LM, Grieco A, Trivedi S, Iakoubov R, Brubaker PL. Novel biological action of the dipeptidylpeptidase-IV inhibitor, sitagliptin, as a glucagon-like peptide-1 secretagogue. Endocrinology 2012;153(2):564-73.

[48] Turco MY, Gardner L, Hughes J, Cindrova-Davies T, Gomez MJ, Farrell L, et al Long-term, hormone-responsive organoid cultures of human endometrium in a chemically defined medium. Nat Cell Biol 2017.

[49] R Core Team. R: a language and environment for statistical computing. R Found Stat Comput 2013

[50] Imai K, Maeda M, Fujiwara H, Kariya M, Takakura K, Kanzaki H, et al. Dipeptidyl peptidase iv as a differentiation marker of the human endometrial glandular cells. Hum Reprod 1992;7(9):1189-94.

[51] Suhorutshenko M, Kukushkina V, Velthut-Meikas A, Altmae S, Peters M, Magi R, et al. Endometrial receptivity revisited: endometrial transcriptome adjusted for tissue cellular heterogeneity. Hum Reprod 2018;33(11):2074-86.

[52] Murakami K, Bhandari H, Lucas ES, Takeda S, Gargett CE, Quenby S, et al. Deficiency in clonogenic endometrial mesenchymal stem cells in obese women with reproductive failure-a pilot study. PLoS ONE 2013;8(12):e82582.

[53] Cloke B, Huhtinen K, Fusi L, Kajihara T, Yliheikkila M, Ho KK, et al. The androgen and progesterone receptors regulate distinct gene networks and cellular functions in decidualizing endometrium. Endocrinology 2008;149(9):4462-74.

[54] Vento-Tormo R, Efremova M, Botting RA, Turco MY, Vento-Tormo M, Meyer KB et al. Single-cell reconstruction of the early maternal-fetal interface in humans. Nature 2018;563(7731):347-53

[55] Wang W, Choi BK, Li W, Lao Z, Lee AY, Souza SC, et al. Quantification of intact and truncated stromal cell-derived factor-1alpha in circulation by immunoaffinity enrichment and tandem mass spectrometry. J Am Soc Mass Spectrom 2014;25 (4):614-25.

[56] Fadini GP, Boscaro E, Albiero M, Menegazzo L, Frison V, de Kreutzenberg S, et al The oral dipeptidyl peptidase-4 inhibitor sitagliptin increases circulating endothelial progenitor cells in patients with type 2 diabetes: possible role of stromalderived factor-1alpha. Diabetes Care 2010;33(7):1607-9.

[57] Schachinger V, Erbs S, Elsasser A, Haberbosch W, Hambrecht R, Holschermann H, et al. Intracoronary bone marrow-derived progenitor cells in acute myocardial infarction. N Engl J Med 2006;355(12):1210-21.

[58] Brenner C, Adrion C, Grabmaier U, Theisen D, von Ziegler F, Leber A, et al. Sitagliptin plus granulocyte colony-stimulating factor in patients suffering from acute myocardial infarction: a double-blind, randomized placebo-controlled trial of efficacy and safety (SITAGRAMI trial). Int J Cardiol 2016;205:23-30.

[59] Santamaria X, Cabanillas S, Cervello I, Arbona C, Raga F, Ferro J, et al. Autologous cell therapy with CD133+ bone marrow-derived stem cells for refractory Asherman's syndrome and endometrial atrophy: a pilot cohort study. Hum Reprod 2016;31(5):1087-96.

[60] Deacon CF, Lebovitz HE. Comparative review of dipeptidyl peptidase-4 inhibitors and sulphonylureas. Diabetes Obes Metab 2016;18(4):333-47.

[61] Boots CE, Bernardi LA, Stephenson MD. Frequency of euploid miscarriage is increased in obese women with recurrent early pregnancy loss. Fertil Steri 2014:102(2):455-9.

[62] Alba M, Sheng D, Guan Y, Williams-Herman D, Larson P, Sachs JR, et al. Sitagliptin $100 \mathrm{mg}$ daily effect on DPP-4 inhibition and compound-specific glycemic improvement. Curr Med Res Opin 2009;25(10):2507-14.

[63] Farag SS, Nelson R, Cairo MS, O’Leary HA, Zhang S, Huntley C, et al. High-dose sitagliptin for systemic inhibition of dipeptidylpeptidase-4 to enhance engraftment of single cord umbilical cord blood transplantation. Oncotarget 2017;8 (66):110350-7. 